\title{
OBSERVATIONAL RECORD \\ EPILITHIC DIATOMS OF THE STREAM OUTFLOW OF WILLIAMS LAKE, HALIFAX, NOVA SCOTIA, WITH NEW RECORDS FOR THE PROVINCE
}

\author{
ALISON R. SHERWOOD*1 AND RONALD J. DECKERT ${ }^{2}$ \\ ${ }^{1}$ Department of Botany, 3190 Maile Way, University of Hawai'i, \\ Honolulu, Hawai'i, U.S.A. 96822 \\ ${ }^{2}$ Department of Biological Sciences, Northern Arizona University, \\ Flagstaff, Arizona, U.S.A. 86011
}

\begin{abstract}
A December 2000 sampling of epilithic diatoms from the outflow of Williams Lake, Halifax County, Nova Scotia, recorded 31 species not previously known from Nova Scotia.

Un échantillonnage effectué en décembre 2000 dans l'émissaire du lac Williams (comté de Halifax) a révélé la présence de 31 espèces de diatomées épilithiques qui n'avaient jamais été mentionnées en Nouvelle-Écosse.
\end{abstract}

\section{Introduction}

From a December 2000 sampling of epilithic diatoms from the outflow of Williams Lake $\left(44^{\circ} 37^{\prime} \mathrm{N}, 63^{\circ} 36^{\prime} \mathrm{W}\right)$, Halifax County, Nova Scotia, the following taxa were recorded. The data are of interest due to the large number of new records $\left(^{*}\right)$ for the province, as determined from a checklist of freshwater diatoms reports from publications such as Blouin et al. (1984), Delorme et al. (1984), Staker \& Hanic (1978) and Vaughan et al. (1982).

\section{Epilithic diatoms identified from the stream outflow of Williams Lake}

Achnanthidium minutissimum (Kütz.) Czarn.*

Actinella punctata Lewis

Aulacoseira granulata (Ehrenb.) Simonsen

Brachysira serians (Bréb. ex Kütz.) Round et D.G. Mann*

B. vitrea (Grun.) R. Ross ex B. Hartley

Cocconeis placentula var. lineata (Ehrenb.) Van Heurck*

Cyclotella comta (Ehrenb.) Kütz.

C. stelligera (Cleve et Grun.) Van Heurck*

Cymbella cesatii (Rabenh.) Grun. ex A.W.F. Schmidt*

Diatoma tenue var. elongatum Lyngb.*

Encyonema caespitosum Kütz.*

E. gracile Rabenh.*

E. silesiacum (Bleisch ex Rabenh.) D.G. Mann*

Eunotia curvata (Kütz.) Lagerst.*

E. curvata var. capitata (Grun.) R.M. Patrick*

E. curvata var. subarcuata (Naeg.) Woodhead et Tweed*

E. incisa W. Sm. ex Greg.

E. parallela Ehrenb.*

* Author to whom correspondence should be addressed. 
E. pectinalis f. minor (Dillwyn) Rabenh.

E. pectinalis var. ventricosa Grun.

E. vanheurckii var. intermedia (Krasske) R.M. Patrick*

Fragilaria acidobiontica Charles*

Frustulia rhomboides var. crassinervia (Bréb.) R. Ross*

Gomphonema acuminatum Ehrenb.*

Gomphonema sp. \#1 PIRLA*

G. gracile Ehrenb. emend. Van Heurck*

G. variostriatum Camburn et Charles*

Neidium affine (Ehrenb.) Pfitz.*

Nitzschia recta Hantzsch*

Pinnularia braunii var. amphicephala (Mayer) Hust.*

P. latevittata Cleve*

P. mesolepta var. angusta Cleve*

Psammothidium marginulatum (Grun.) Bukht. et Round*

Rossithidium linearis (W. Sm.) Round et Bukht.*

Sellophora rectangularis (W. Greg.) Lange-Bert. et Metzeltin ex Lange-Bert.*

Semiorbis hemicyclus (Ehrenb.) R.M. Patrick*

Stauroneis anceps f. linearis (Ehrenb.) Hust.*

Surirella biseriata var. bifrons f. amphioxys (W. Sm.) Hust.*

Tabellaria flocculosa (Roth) Kütz.

T. quadriseptata Knuds.

\section{References}

Blouin AC, Lane PA, Collins TM, Kerekes JJ (1984) Comparison of plankton-water chemistry relationships in three acid stressed lakes in Nova Scotia, Canada. Int Revue Gesamten Hydrobiol 69:819-841

Delorme LD, Esterby SR, Duthie H (1984) Prehistoric pH trends in Kejimkujik Lake, Nova Scotia. Int Revue Gesamten Hydrobiol 69:41-55

Staker RD, Hanic LA (1978) A literature review of the freshwater algae of the Canadian Atlantic provinces, Quebec, Maine and New Hampshire. Proc NS Inst Sci 28:171-180 Vaughan HH, Underwood JK, Ogden JG (1982) Acidification of Nova Scotian lakes: response of diatom assemblages in the Halifax area. Water Air Soil Pollut 18:353-361 